\title{
Validity Evaluation of Estimating Missed or Scratched Questions in Computer-Based Implementation of Conners’ Attention Questionnaire*
}

\author{
Farnaz Ghassemi \\ ICSS (Institute for \\ Cognitive Science Studies), \\ Amirkabir University of \\ Technology, Tehran, Iran
}

\author{
Mehdi Tehrani-Doost \\ ICSS (Institute for Cognitive \\ Science Studies), Tehran \\ University of Medical \\ Science, Tehran, Iran
}

\author{
Mohammad Hassan \\ Moradi \\ Amirkabir University of \\ Technology, Tehran, Iran
}

\author{
Vahid Abootalebi \\ Yazd University, \\ Yazd, Ira
}

\begin{abstract}
The probability of missing the questions by patients' suffering from ADHD (attention deficit hyperactivity disorder) is high and can disturb the evaluation of questionnaire. In this case, the operator should complete the missed answers by considering their estimations to evaluate the questionnaire. This estimation can be calculated by averaging the answer of other questions in that subscale. The goal of this study is to evaluate the validity of this estimation in the screening version of the CAARS (Conners adult ADHD rating scale) questionnaire. The results indicated that although the difference between original and estimated values is significant for some particular questions individually, evaluating the estimation did not show any significant differences in the whole groups. Considering this fact, if the missed question is a critical one, more considerations shall be paid.
\end{abstract}

Keywords: ADHD (attention deficit hyperactivity disorder), CAARS (Conners adult ADHD rating scale), Kruskal-Wallis test, multiple comparison procedure, PCA (principal component analysis)

\section{Introduction}

ADHD (attention deficit hyperactivity disorder) is primarily thought to be a problem limited to youth. However, researchers have found that ADHD is often a chronic condition which persists into adulthood in 30\% to $70 \%$ of the patients (Conners, Erhardt, \& Sparrow, 1999; Searight \& Hermann, 2010; Vollmer, 1998; Wender,

\footnotetext{
*Acknowledgment: The authors are thankful to the ICSS (Institute for Cognitive Science Studies) for prearranging of CAARS questionnaire. They appreciate Dr. Anahita Khorrami for her help in conducting the test for some participants. The authors also thank all participants for their contribution in this study.

This paper is the completed version of a conference paper \& internal journal cited as:"Validity evaluation of estimating missed or scratched questions in computer-based implementation of Conner's attention questionnaire”, Farnaz Ghassemi, Mohammad Hassan Moradi, Mehdi Tehrani-Doost, Vahid Abootalebi, 3rd International Conference of Cognitive Science, Iran 3-5 March 2009; "Evaluation of estimating missed answers in conners adult ADHD rating scale (Screening Version)", Farnaz Ghassemi, Mohammad Hassan Moradi, Mehdi Tehrani-Doost, Vahid Abootalebi, Iranian Journal of Psychiatry, vol. 5, pp. 108-112, 2010.

Farnaz Ghassemi, Ph.D., assistant professor, ICSS (Institute for Cognitive Science Studies), Department of Biomedical Engineering, Amirkabir University of Technology.

Mehdi Tehrani-Doost, Child and Adolescent Psychiatrist, associate professor, ICSS (Institute for Cognitive Science Studies), Department of Psychiatry, Tehran University of Medical Science.

Mohammad Hassan Moradi, Ph.D., associate professor, Department of Biomedical Engineering, Amirkabir University of Technology.

Vahid Abootalebi, Ph.D., assistant professor, Department of Electrical and Computer Engineering, Yazd University.
} 
1998). ADHD is characterized as having inattentiveness, hyperactivity and impulsivity. Although, in children and adolescents, this disorder is more common in boys, adult ADHD appears to be more equally balanced between men and women (Simon, Czobor, Balint, Mészáros, \& Bitter, 2009) and it appears to decrease with age (Searight \& Hermann, 2010).

In addition to the core symptoms of ADHD, adults' subject to this disorder have been found to be at risk for a variety of other problems and conditions. For example, ADHD adults have been found to be at risk for lower levels of educational and occupational attainment, employment instability, substance abuse and antisocial behavior (Conners et al., 1999). It is estimated that between $1 \%$ and $7 \%$ of the adult population experiences ADHD symptoms (Searight \& Hermann, 2010; Vollmer, 1998; Wender, 1998).

Up to now, different studies have been carried out regarding various types of attention and their relations with brain activities. There are activation states of cerebral cortex that affect the ability to process information where the activation itself contains no specific information. These activation states can be tonic or phasic and may be relatively global or more localized. Terms that have been used to describe these states include arousal, alertness, vigilance and attention. Unfortunately, no term is ideal to describe these states of cortical activation since most terms are in broad use with varied associations and there are not perfect physiological markers (Oken, Salinsky, \& Elsas, 2006; Parasuraman, 1998; Posner \& Petersen, 1990).

The four most commonly used self-report measures for ADHD are the CAARS (Conners adult ADHD rating scale) (Conners, Erhardt, \& Sparrow, 1999), the Wender rating scale (Wrad, Wender, \& Reimherr, 1993), the Copeland symptom checklist (Copeland, 1991) and the Brown scale (Brown, 1996). These screening tools should not be used for diagnostic purposes, as inattention, impulsivity and volatile moods are features of several other psychiatric conditions. The screening forms are useful when a quick screen for ADHD symptoms based on the fourth edition of the DSM-IV (diagnostic and statistical manual of mental disorders) (American Psychiatric Association, 1994) is required.

A main problem in self-report measures is the high probability of missing the questions by patients suffering from ADHD which can disturb the evaluation of questionnaire. In this case, to evaluate the questionnaire, the operator should complete the missed answers. Therefore, the missed answer should be estimated through averaging the answer of other questions on that subscale and inserting the result for the missed answer.

The CAARS is one of the four mentioned questionnaires in evaluating ADHD. It is designed to assess symptoms and behaviors related to ADHD in adults and causes development in the assessment of the psychopathology and problem behaviors associated with adults ADHD. The goal of this study is to evaluate the validity of estimating missed or scratched answers in the screening version of the CAARS questionnaire and also extract its principal components.

\section{Method and Materials}

\section{Conners' Questionnaire}

The CAARS questionnaire is a suitable instrument for reporting symptoms and behaviors related to ADHD on adults aged 18 and up. Both self-report and observer forms utilize a four-point, Likert-style format in which respondents are asked to rate items pertaining to their behavior/problems. Each mark stands for: 0: "Not at all, never”; 1: “Just a little, once in a while”; 2: "Pretty much, often”; 3: "Very much, very frequently”.

The self-report screening form CAARS-S: SV (CAARS-Self report: Screening Version) which is used in 
this study, includes 30 items that assess ADHD symptoms, according to the DSM-IV criteria (American Psychiatric Association, 1994). The subscales derived from the questionnaire are: (1) inattentive (9 items); (2) hyperactive-impulsive (9 items); (3) total ADHD symptoms; and (4) ADHD index (12 items).

Interpretation of the CAARS requires a general understanding of the nature of ADHD symptoms across the life span. The CAARS should be interpreted based on an analysis of where a particular individual's scores fall with respect to the CAARS population norms. For example, an individual with a $t$-score above 70 on the ADHD index is likely to have significant levels of symptoms that may meet diagnostic criteria, such as in the DSM-IV (Conners et al., 1999).

\section{Participants}

We asked 600 students at four universities to complete the questionnaire which had been sent to them via e-mail. We also distributed 120 paper-forms of the questionnaire among the students. A total number of 530 questionnaires were returned among which 130 participants' answers were incomplete or scratched and 400 questionnaires were filled out completely. This indicates the importance and need of proper estimation of missed or scratched answers in the questionnaire. Figure 1 demonstrates the characteristics of the participants in age, gender and education. Men and women are almost equally distributed and most of the participants are graduated. Since financial incentives affect the attention level (Oken et al., 2006), subjects were not paid to participate in the experiment. In generalization of the results, it should be taken into account that the study was performed on a population of university students.

\section{Statistical Analysis and Validity Evaluation}

Software version of the questionnaire was prepared and used which has the benefits of fast evaluation of the questionnaire, increasing the accuracy, simple dispatching to each participant by e-mail and simplicity of constructing the database for statistical examinations. All statistical tests and calculations were performed with "Matlab 2009" software.

Lilliefors test was used to evaluate whether the distribution of the sample was normal. The Lilliefors test is a two-sided goodness-of-fit test suitable when a fully-specified null distribution is unknown and its parameters must be estimated, i.e., it is a Kolmogorov-Smirnov test with unknown null distribution. The raw scores of the CAARS were converted to $t$-scores to eliminate the gender and age effects. The $t$-scores used in CAARS are linear ones which do not change the actual distributions of the variables (1). Therefore, the variables which are not normally distributed in the raw data will continue to be distributed in this way after being converted to $t$-scores.

To score the questionnaire, missed or scratched answers are estimated by calculating the average of other answers in that subscale. For example, the first question belongs to inattention subscale which contains nine questions. If this question is missed, the average of the other eight questions will be considered as the estimation of its answer. To investigate the validity of the estimated missed or scratched answers, we assumed each question was missed individually and estimated its answer. Then, we compared the real answers with their calculated estimations for each question to find any significant difference.

We used Kruskal-Wallis test to investigate the veracity of this approach. This test is a non-parametric version of ANOVA (analysis of variance). For each question, an individual test was performed to evaluate the difference between the original answer and its estimation (30 tests). Considering that there is a possibility for each of the questions to be missed, the significance of the difference should be evaluated in the whole subscale, 
so we used the MCP (multiple comparison procedure) to evaluate the significance of the differences between the real and estimated answers in each subscale. Since the chance of incorrectly finding, a significant difference would increase with the number of comparisons, using the MCP can provide an upper bound on the probability that any comparison can be incorrectly found significant.

In some questionnaires, the number of questions (dimension of the input vector) is large, but the questions are highly correlated (redundant). It is useful in this situation to reduce the dimension of the input vectors. An effective procedure for performing this operation is PCA (principal components analysis) (Jolliffe, 2002). This technique orthogonalizes the components of the input vectors, which means that transforming a number of possibly correlated variables into a smaller number of uncorrelated variables called principal components. This method was used for extracting principal components of the CAARS questionnaire (Ghassemi, Moradi, Tehrani-Doost, \& Abootalebi, 2010).

\section{Results}

Figure 1 demonstrates the characteristics of participants. The average age of the participants was $24.4 \pm 5.9$ years and $47.75 \%$ of them were male. In this population, $68 \%$ were graduated and $14 \%$ were under-graduated.
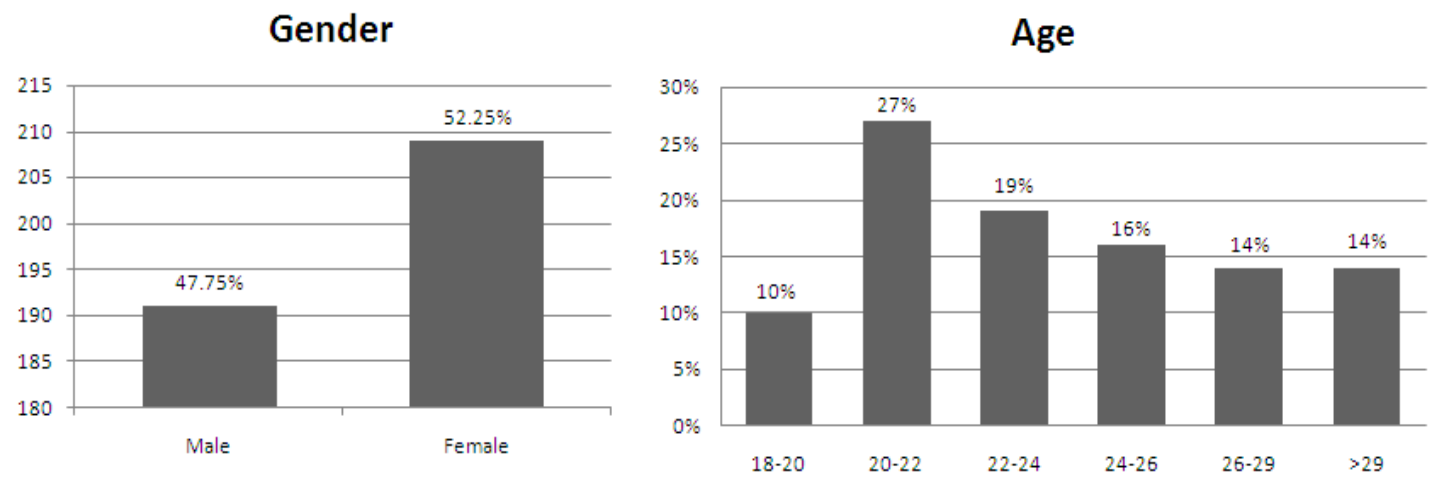

\section{Education}

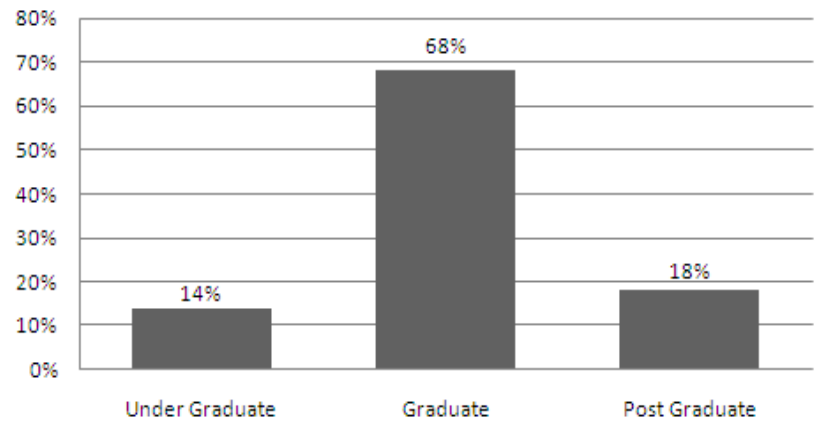

Figure 1. The characteristics of subjects' distribution in age, gender and education.

The distribution of $t$-scores in the statistical population for each subscale is shown in Figure 2. The $t$-scores used with CAARS have a mean of 50 and a standard deviation of ten. Values around 50 indicate that the participant is in the average range in that subscale, whereas higher $t$-scores represent a problem and lower $t$-scores suggest that the participant does not present particular symptoms. 


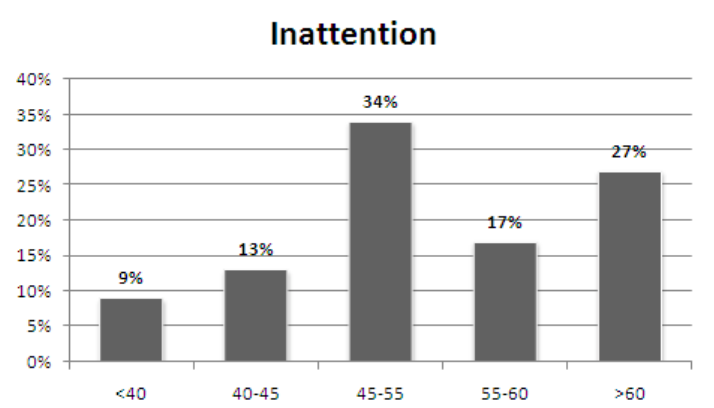

ADHD

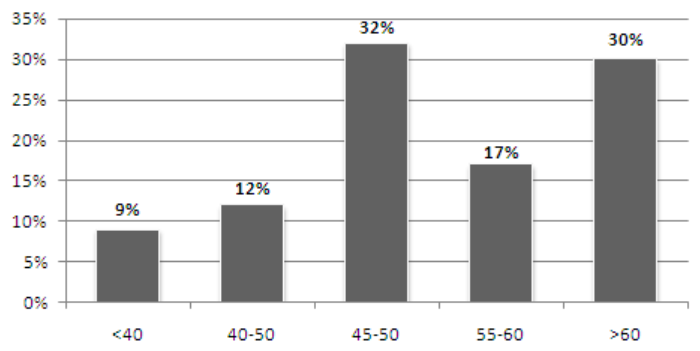

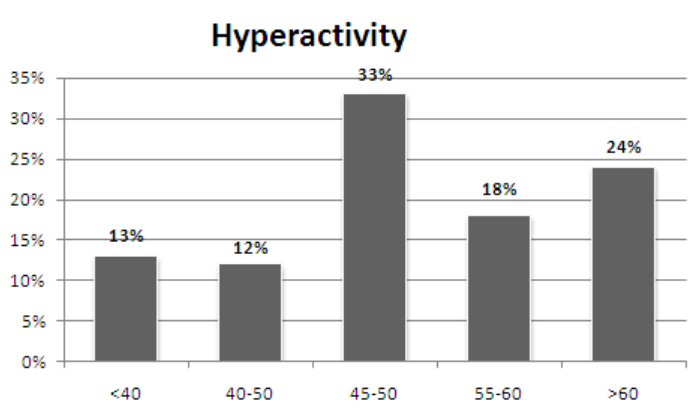

ADHD Index

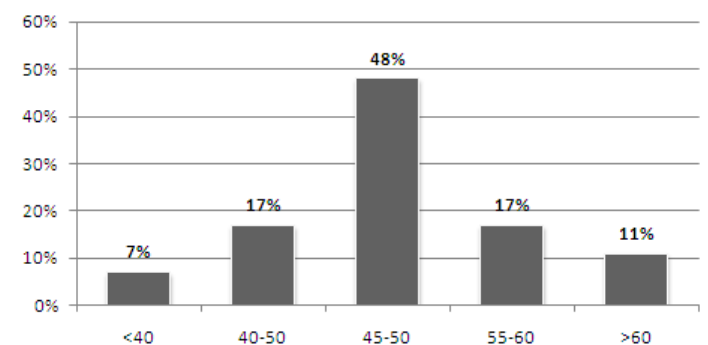

Figure 2. The distribution of subjects' $t$-scores in four subscales (inattention, hyperactivity, ADHD and ADHD index).

Table 1

The Results of Kruskal-Wallis

\begin{tabular}{|c|c|c|c|c|c|c|c|c|c|}
\hline Q No. & Group & $P$-value & Group & $P$-value & Q No. & Group & $P$-value & Group & $P$-value \\
\hline 1 & $\mathrm{~A}$ & 0.151 & $\mathrm{C}$ & 0.919 & 16 & $\mathrm{D}$ & $0^{*}$ & & \\
\hline 2 & B & 0.416 & $\mathrm{C}$ & 0.154 & 17 & B & 0.786 & $\mathrm{C}$ & 0.019 \\
\hline 3 & $\mathrm{D}$ & $0^{*}$ & & & 18 & A & 0.092 & $\mathrm{C}$ & 0.012 \\
\hline 4 & B & $0^{*}$ & $\mathrm{C}$ & $0^{*}$ & 19 & $\mathrm{D}$ & 0.647 & & \\
\hline 5 & $\mathrm{D}$ & $0.001^{*}$ & & & 20 & A & $0^{*}$ & $\mathrm{C}$ & $0^{*}$ \\
\hline 6 & B & 0.724 & $\mathrm{C}$ & 0.2231 & 21 & $\mathrm{~B}$ & 0.56 & $\mathrm{C}$ & 0.015 \\
\hline 7 & $\mathrm{D}$ & $0^{*}$ & & & 22 & $\mathrm{D}$ & $0^{*}$ & & \\
\hline 8 & B & 0.1787 & $\mathrm{C}$ & $0.003^{*}$ & 23 & $\mathrm{D}$ & $0^{*}$ & & \\
\hline 9 & A & 0.726 & $\mathrm{C}$ & 0.366 & 24 & B & 0.067 & $\mathrm{C}$ & $0^{*}$ \\
\hline 10 & $\mathrm{D}$ & $0^{*}$ & & & 25 & A & 0.098 & $\mathrm{C}$ & $0.006^{*}$ \\
\hline 11 & $\mathrm{D}$ & 0.773 & & & 26 & $\mathrm{~B}$ & $0^{*}$ & $\mathrm{C}$ & $0^{*}$ \\
\hline 12 & $\mathrm{~A}$ & 0.726 & $\mathrm{C}$ & 0.533 & 27 & $\mathrm{D}$ & $0^{*}$ & & \\
\hline 13 & A & $0^{*}$ & $\mathrm{C}$ & $0^{*}$ & 28 & A & $0^{*}$ & $\mathrm{C}$ & $0^{*}$ \\
\hline 14 & $\mathrm{D}$ & $0^{*}$ & & & 29 & A & $0.001^{*}$ & $\mathrm{C}$ & $0^{*}$ \\
\hline 15 & B & $0^{*}$ & $\mathrm{C}$ & $0^{*}$ & 30 & $\mathrm{D}$ & $0^{*}$ & & \\
\hline
\end{tabular}

Notes. P-values less than 0.01 which are marked with “*” indicate significant difference between original answer and its estimation.

$T$-scores distribution in the statistical population indicated that in "Inattention" group (A), 34\% of the participants were in the average range (regarding their age and gender), $27 \%$ were very much above the average having high levels of symptoms that could meet the diagnostic criteria for ADHD and 9\% were much below the average. In "Hyperactivity" group (B), the average range contained 33\% of the participants. Twenty-four percent were very much above the average and $13 \%$ were much below the average. In "ADHD” group (C), $32 \%$ of the statistical population was in the average range, 30\% was very much above the average and $9 \%$ was quite below the average, whereas $17 \%$ and $12 \%$ were slightly above and below the average, respectively. In 
"ADHD Index" group (D), $48 \%$ of the statistical population was in the average range, $11 \%$ was very much above and $7 \%$ much below the average.

The result of the Lilliefors test indicated that the distribution of $t$-scores and, consequently, the distribution of raw scores were not normal. Thus, non-parametric tests were used for statistical evaluations.

Table 1 demonstrated the results of Kruskal-Wallis tests which define the questions included in each subscale and express the obtained $P$-value. $P$-values less than 0.01 indicated significant differences between original answer and its estimation. Results showed that a significant difference ( $d f=399, P<0.01)$ exists between the original and estimated answers for some questions. Therefore, the substitution method was not appropriate for their estimation.

Table 2

The Results of Principal Component Analysis

\begin{tabular}{|c|c|c|c|c|c|c|c|c|c|c|c|c|c|c|}
\hline Component 1 & 2 & 3 & 4 & 5 & 6 & 7 & 8 & 9 & 10 & 11 & 12 & 13 & 14 & 15 \\
\hline Eigen value 6.765 & 3.156 & 2.044 & 1.438 & 1.286 & 1.19 & 1.164 & 1.014 & 0.981 & 0.851 & 0.78 & 0.764 & 0.749 & 0.718 & 0.699 \\
\hline $\begin{array}{l}\text { Percentage } \\
\text { of variance }\end{array} 22.549$ & 10.521 & 6.814 & 4.794 & 4.288 & 3.968 & 3.879 & 3.381 & 3.270 & 2.835 & 2.602 & 2.546 & 2.495 & 2.394 & 2.392 \\
\hline $\begin{array}{l}\text { Cumulative } \\
\text { percentage }\end{array} 22.549$ & 33.070 & 39.884 & 44.678 & 48.9665 & 52.9345 & 56.813 & 60.194 & 63.464 & 66.299 & 68.901 & 71.447 & 73.943 & 76.337 & 78.666 \\
\hline Component 16 & 17 & 18 & 19 & 20 & 21 & 22 & 23 & 24 & 25 & 26 & 27 & 28 & 29 & 30 \\
\hline lue 0.672 & 0.629 & 0.565 & 0.547 & 0.515 & 0.464 & 0.445 & 0.416 & 0.318 & 0.371 & 0.354 & 0.285 & 0.275 & 0.253 & 0.233 \\
\hline $\begin{array}{ll}\text { age } & 2.392 \\
\text { ince } & \end{array}$ & 2.097 & 1.882 & 1.823 & 1.716 & 1.547 & 1.483 & 1.377 & 1.270 & 1.237 & 1.178 & 0.950 & 0.915 & 0.842 & 0.776 \\
\hline $\begin{array}{l}\text { umulative } 80.905 \\
\text { ercentage }\end{array}$ & 3.00 & 0400 & 868 & 38.42 & 89.97 & & 9287 & 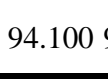 & & $6-5$ & & & & \\
\hline
\end{tabular}

Table 3

The Component Matrix

\begin{tabular}{lcccccccccccccccc}
\hline & $\mathrm{Q} 1$ & $\mathrm{Q} 2$ & $\mathrm{Q} 3$ & $\mathrm{Q} 4$ & $\mathrm{Q} 5$ & $\mathrm{Q}$ 6 & Q 7 & Q 8 & Q 9 & Q 10 & Q 11 & Q 12 & Q 13 & Q 14 & Q 15 \\
\hline 1 & 0.397 & 0.358 & 0.127 & 0.345 & 0.332 & 0.514 & 0.459 & 0.419 & 0.644 & 0.416 & 0.542 & 0.48 & 0.454 & 0.479 & 0.479 \\
2 & -0.115 & 0.371 & 0.767 & 0.413 & 0.169 & 0.39 & 0.35 & 0.235 & -0.191 & -0.38 & 0.194 & -0.191 & -0.184 & -0.039 & -0.322 \\
3 & -0.027 & 0.35 & -0.246 & -0.193 & 0.296 & -0.181 & 0.276 & 0.242 & -0.267 & -0.253 & -0.167 & -0.316 & 0.28 & 0.254 & 0.373 \\
4 & 0.463 & 0.011 & 0.117 & -0.056 & -0.384 & 0.046 & -0.300 & -0.003 & -0.075 & -0.302 & -0.170 & 0.147 & 0.516 & -0.155 & 0.015 \\
5 & 0.129 & -0.155 & 0.013 & -0.114 & 0.004 & 0.028 & 0.143 & -0.175 & -0.124 & 0.15 & 0.243 & -0.518 & -0.071 & -0.45 & 0.119 \\
6 & 0.091 & -0.362 & -0.123 & -0.104 & 0.346 & 0.041 & -0.002 & 0.388 & -0.142 & 0.038 & 0.409 & 0.097 & 0.043 & 0.042 & -0.006 \\
7 & -0.503 & 0.042 & 0.034 & -0.223 & -0.276 & -0.270 & -0.173 & 0.041 & 0.029 & 0.088 & 0.222 & 0.148 & -0.244 & 0.117 & 0.004 \\
8 & 0.232 & 0.195 & -0.016 & 0.029 & 0.407 & -0.073 & 0.281 & -0.344 & 0.077 & 0.064 & -0.084 & 0.320 & 0.051 & -0.016 & -0.107 \\
\hline & $\mathrm{Q} 16$ & $\mathrm{Q} 17$ & $\mathrm{Q} 18$ & $\mathrm{Q} 19$ & $\mathrm{Q} 20$ & $\mathrm{Q} 21$ & $\mathrm{Q} 22$ & $\mathrm{Q} 23$ & $\mathrm{Q} 24$ & $\mathrm{Q} 25$ & $\mathrm{Q} 26$ & $\mathrm{Q} 27$ & $\mathrm{Q} 28$ & $\mathrm{Q} 29$ & $\mathrm{Q} 30$ \\
\hline 1 & 0.108 & 0.588 & 0.468 & 0.693 & 0.481 & 0.474 & 0.466 & 0.013 & 0.551 & 0.321 & 0.631 & 0.448 & 0.482 & 0.645 & 0.648 \\
2 & 0.622 & -0.156 & 0.172 & -0.186 & 0.177 & -0.118 & 0.662 & 0.232 & -0.131 & 0.255 & -0.335 & 0.215 & -0.281 & -0.171 & -0.362 \\
3 & -0.453 & 0.057 & 0.068 & -0.141 & 0.444 & -0.233 & -0.265 & 0.221 & -0.197 & 0.371 & 0.065 & 0.359 & -0.2 & -0.266 & -0.006 \\
4 & 0.201 & 0.060 & 0.068 & -0.141 & 0.444 & -0.233 & -0.265 & 0.221 & -0.197 & 0.371 & 0.065 & 0.359 & -0.200 & -0.266 & -0.006 \\
5 & 0.132 & 0.190 & 0.265 & -0.006 & 0.068 & 0.105 & 0.031 & 0.16 & -0.21 & -0.328 & 0.137 & 0.164 & 0.282 & -0.318 & 0.224 \\
6 & -0.162 & 0.035 & 0.359 & -0.155 & -0.138 & -0.291 & 0.076 & 0.116 & -0.105 & 0.093 & -0.165 & -0.295 & 0.092 & 0.127 & -0.081 \\
7 & 0.045 & -0.096 & 0.140 & -0.027 & 0.209 & -0.228 & 0.006 & 0.393 & 0.097 & -0.132 & -0.075 & 0.329 & 0.226 & 0.227 & -0.010 \\
8 & -0.088 & 0.000 & -0.098 & -0.085 & 0.107 & -0.130 & -0.059 & 0.159 & -0.153 & -0.350 & -0.235 & 0.091 & 0.140 & 0.243 & -0.160 \\
\hline
\end{tabular}

As Table 1 demonstrates, this estimation is significantly different from the real value for questions: (A) 13 , 20, 28, 29 (inattentive subscale); (B) 4, 15, 26 (hyperactive/impulsive subscale); (C) 4, 13, 15, 18, 20, 26, 28, 


\section{9 (ADHD subscale); (D) 3, 7, 10, 14, 16, 22, 23, 27, 30 (ADHD index).}

The results of MCP showed that the estimation, when evaluated in the whole subscale, was not significantly different $(\alpha=0.001)$ from the real value in each subscale.

Table 2 indicates the results of PCA which transforms a number of possibly correlated variables into a smaller number of uncorrelated variables. This table expresses the obtained Eigen values, percents of variance and cumulative percents of the components. As it can be seen, eight components had Eigen values greater than one and can be extracted. These components contained $60 \%$ of variability of all items. The component matrix is demonstrated in Table 3 which shows the effect of every question on each of eight obtained components. For example, the first component was mostly affected by questions 9, 19, 26, 29 and 30.

\section{Discussion and Conclusions}

ADHD is a common problem in adults which can be evaluated using some self-report questionnaires including the CAARS. A main problem in self-report measures is the high probability of missing some questions by ADHD patients which can interfere with scoring of the questionnaire. In this case, the operator should complete the missed answers to score the questionnaire. This study is dedicated to evaluate the estimation of the missed answers in the self-report screening version of CAARS completed by 400 university students.

The Kruskal-Wallis results revealed a significant difference $(P$-value $<0.01)$ between the real and estimated values for some particular questions: (1) four questions in the "inattention" subscale; (2) three in the "hyperactive/impulsive" subscale; (3) eight in the "ADHD” subscale; and (4) nine in the "ADHD index" subscale.

It should be noticed that the estimation of a particular question in A or B subscale was obtained by averaging eight other questions in that subscale, while its estimation in $\mathrm{C}$ subscale was obtained by averaging 17 other questions.

Evaluation of each question individually indicated that only the estimation of the answers in ten questions could be valid (numbers 1, 2, 6, 9, 11, 12, 17, 18, 19 and 21). Therefore, when a high percentage of the participants in one study has missed a particular question, it means that the estimation should not be used for the critical questions mentioned in Table 1 and the incomplete questionnaires should be removed from the study. However, the results of MCP, when evaluated in the whole subscales, indicated that the estimations did not differ significantly from their real values in four subscales, i.e., the result of the whole subscales did not change with this estimation.

The obtained results can emphasize on the fact that this questionnaire is mainly designed for screening purposes and this estimation does not change the results of subscales when a question is missed randomly. Therefore, if many of the participants missed a critical question, this substitution would not be valid and more considerations should be paid in such cases.

Eight principal components are extracted by means of PCA which transforms a number of possibly correlated variables into a smaller number of uncorrelated variables called principal components. The first component accounts for as much of the variability in the data as possible and each succeeding component accounts for as much of the remaining variability as possible.

The novelty of this study is in the evaluation method of estimation. Regarding similar studies, PCA is used as a factor analysis method in translated versions of ADHD questionnaires (Caci, Bayle, \& Bouchez, 2008; Erhart, Dopfner, \& Ravens-Sieberer, 2008; Du, Kou, \& Coghill, 2008). In the French version of ASRS (ADHD 
self-report scale), self-report adult questionnaire was considered and factor analyses retrieved the original two-factor structure although impulsivity items clearly load on a specific third factor in students only (Caci et al., 2008). Also, German and Chinese children were respectively investigated (Erhart et al., 2008; Du et al., 2008). Psychometric properties of CAARS and FBB-HKS questionnaires were compared for the former and both instruments' scores showed reliability as well as factorial and convergent/discriminant validity (Erhart et al., 2008). For the latter, mixed findings with respect to the psychometric properties of the Chinese translation of the SDQ were reported (Du et al., 2008). Considering adult Iranian population, one study had reported the prevalence of 3.7\% for ADHD in a group of 244 university students, but no estimation or validity evaluation was investigated (Arabgol, Hayati, \& Hadid, 2004).

Briefly, although the Kruskal-Wallis results revealed that the difference between the original and estimated values is significant for some particular questions individually, the results of MCP, when evaluated in the whole group, indicated that the estimations do not differ significantly from their real values in any of the four subscales, i.e., the result of the whole group will not change with this estimation.

\section{References}

American Psychiatric Association. (1994). Diagnostic and statistical manual of mental disorders (4th ed.).

Arabgol, F., Hayati, M., \& Hadid, M., (2004). Prevalence of attention: Deficit/hyperactivity disorder in a group of university students. Advances in Cognitive Science, 6, 73-78.

Brown, T. E. (1996). Brown attention deficit disorders scales manual. San Antonio, T. X.: Psychological Corporation.

Caci, H., Bayle, F. J., \& Bouchez, J. (2008). Adult ADHD: Translation and factor analyses of the ASRS-1.1 symptom checklist. Retrieved from http://www.pagesperso-orange.fr/herve.caci/Comm/assets/Poster\%20ASRS\%20AEP2008.pdf

Conners, C. K., Erhardt, D., \& Sparrow, E. (1999). Conners' adult ADHD rating scales (CAARS). New York: Multi-Health Systems, Inc..

Copeland, E. D. (1991). Medications for attention disorders and related medical problems. Atlanta, G. A.: SPI Press.

Du, Y., Kou, J., \& Coghill, D. (2008). The validity, reliability and normative scores of the parent, teacher and self report versions of the strengths and difficulties questionnaire in China. Child Adolesc Psychiatry Ment Health, 2, 8.

Erhart, M., Dopfner, M., \& Ravens-Sieberer, U. (2008). Psychometric properties of two ADHD questionnaires: Comparing the Conners' scale and the FBB-HKS in the general population of German children and adolescents-Results of the BELLA study. Eur Child Adolesc Psychiatry, 115, 106-115.

Ghassemi, F., Moradi, M. H., Tehrani-Doost, M, \& Abootalebi, V. (2010). Evaluation of estimating missed answers in Conners adult ADHD rating scale (screening version). Iranian Journal of Psychiatry, 5, 108-112.

Jolliffe, I. T. (2002). Principal component analysis. In Series: Springer series in statistics (2th ed.). New York: Springer.

Mannuzza, S., Klein, R. G., Bonagura, N., Malloy, P., Giampino, T. L., \& Addalli, K. A. (1991). Hyperactive boys almost grown up: V. Replication of psychiatric status. Arch Gen Psychiatry, 48, 77-83.

Oken, B. S., Salinsky, M. C., \& Elsas, S. M. (2006). Vigilance, alertness, or sustained attention: Physiological basis and measurement. Clin Neurophysiol, 117, 1885-1901.

Parasuraman, R. (1998). The attentive brain. Cambridge, M. A: The MIT Press.

Posner, M., \& Petersen, S. (1990). The attentional system of the human brain. Ann Rev Neuroscience, 42, 13-25.

Searight, H. R., \& Hermann R. (2010). Adult attention deficit hyperactivity disorder. In J. M. Bruke (section editor), \& T. L. Schwenk (deputy editor) (Eds.), Up to date. Retrieved January 18, 2010, from http://www.uptodate.com/home/about/ index.html

Simon V., Czobor P., Balint S., Mészáros A., \& Bitter I. (2009). Prevalence and correlates of adult attention-deficit hyperactivity disorder: Meta-analysis. British Journal of Psychiatry, 194, 204-211.

Vollmer, S. (1998). ADHD: It's not just in children. Family Practice Recertification, 20, 45-67.

Wender, P. (1998). Attention-deficit hyperactivity disorder in adults. Psychiatric Clinics of North America, 21, 761-774.

Ward, M. F., Wender, P. H., \& Reimherr, F. W. (1993). The Wender Utah rating scale: An aid in the retrospective diagnosis of childhood attention deficit hyperactivity disorder. American Journal of Psychiatry, 150, 885-890. 